\title{
XIX.-Organic Derivatives of Silicon. Part IV. The Sulphonation of Benzylethylpropylsilicyl Oxide and of Benzylethyldipropylsilicane.
}

\section{By Herbert Marsden, B.Sc. (Vict.), and Frederic Stanley KIPPING.}

Phenylbenzylethylpropylsilicane is hydrolysed by hot sulphuric acid giving benzene and benzylethylpropylsilicol (Kipping, Trans., $1907,91,212)$; the latter undergoes sulphonation, yielding an acid which was regarded as the disulphonic derivative of benzylethylpropylsilicyl oxide and to which the constitution

$$
\mathrm{SO}_{3} \mathrm{H} \cdot \mathrm{C}_{6} \mathrm{H}_{4} \cdot \mathrm{CH}_{2} \cdot \mathrm{SiEtPr} \cdot \mathrm{O} \cdot \mathrm{SiEtPr} \cdot \mathrm{CH}_{2} \cdot \mathrm{C}_{6} \mathrm{H}_{4} \cdot \mathrm{SO}_{3} \mathrm{H}
$$

was assigned. Subsequently (Kipping, Trans., 1907, 91, 727) this same sulphonic acid was obtained by the sulphonation of benzylethylpropylsilicol, prepared directly by decomposing benzylethylpropylsilicyl chloride with water.

As this sulphonic acid is the only compound containing an asymmetric silicon group which, so far, has been resolved, and as the evidence that it was derived from the oxide and not from the silicol was, perhaps, not quite conclusive, we have made some further experiments in order to settle the matter.

For this purpose we examined the product of high boiling point which is formed together with benzylethylpropylsilicol when benzylothylpropylsilicyl chloride is decomposed with water, and found, as was indeed expected (loc. cit., p. 726), that it consisted of the oxide $(\mathrm{BzEtPrSi})_{2} \mathrm{O}$; this compound was then sulphonated with sulphuric acid and also with chlorosulphonic acid. In both cases the product was a mixture of acids, from which the isolation of a pure compound proved to be a task of some difficulty; ultimately, however, we obtained from both preparations an acid (in the form of its $l$-menthylamine salt) which was found to be identical with that previously described as sulphobenzylpropylsilicyl oxide.

Now as benzylethylpropylsilicyl oxide and other oxides of like 
nature are relatively very stable, whereas benzylethylpropylsilicol and other silicols are unstable and pass readily into the corresponding oxides, it seems extremely improbable that the oxide in question would be converted into the silicol during the process of sulphonation or subsequent treatment, and therefore the sulphonic acid may be regarded as derived from the oxide; as, moreover, this conclusion is fully borne out by the results of numerous analyses of the $l$-menthyla mine salt which have been made in the course of these experiments, there is now not much room for doubt that the optically active silicon compounds recently described (loc. cit., p. 234) are derivatives of benzylethylpropylsilicyl oxide.

The resolution of the sulphonic derivative of benzylmethylethylpropylsilicane, BzMeEtPrSi, and of other compounds containing only one asymmetric silicon group having so far been unsuccessful, we studied the sulphonation of phenylbenzylethylpropylsilicane,

\section{$\mathrm{PhBzEtPrSi}$,}

with chlorosulphonic acid, in the hope that this agent would not have the hydrolysing action of sulphuric acid; unfortunately, however, we were unable to prepare the simple sulphonic derivative of this silicohydrocarbon, since the phenyl group was eliminated previous to, or during, sulphonation, and the same acid was obtained as by the use of sulphuric acid. The great difference in the behaviour of the phenyl and benzyl groups when combined with silicon is again shown by these results, and it may be noted that in these, as in other experiments, the phenyl radicle alone seems to be easily displaced.

This paper also contains an account of the investigation and utilisation of certain by-products which had accumulated in the preparation of silicon derivatives. Thus from a mixture of benzylethylsilicon dichloride and benzylethylpropylsilicyl chloride, obtained in the preparation of the latter, we prepared benzylethyldipropylsilicane, $\mathrm{BzEtPr} \mathrm{r}_{2} \mathrm{Si}$, and sulphonated this silico-hydrocarbon in order to compare the acid with the $d l$-derivative of benzylmethylethylpropylsilicane (loc. cit., p. 731). Although one of these sulphonic derivatives is externally compensated, whereas the other is not, there is very great similarity in properties even when they are combined with an asymmetric base; the l-menthylamine salt of benzylethyldipropylsilicanesulphonic acid, for example, is indistinguishable by inspection from the corresponding salt of $d l$-benzylmethylethylpropylsilicanesulphonic acid and the resemblance in all ordinary properties is very striking; the cinchonidine and quinine salts of the two acids are also very similar, and the results of the comparison point strongly to the conclusion that these derivatives of the $d l$-acid are mere mixtures and not definite partially racemic salts.

Diphenylethylsilicyl chloride, $\mathrm{Ph}_{2} \mathrm{EtSiCl}$, was isolated from a by- 
product obtained in the preparation of phenylethylsilicon dichloride (loc. cit., p. 215), but the corresponding silicol could not be obtained in a pure state owing to the readiness with which it passes into diphenylethylsilicyl oxide, $\left(\mathrm{Ph}_{2} \mathrm{EtSi}\right)_{2} \mathrm{O}$, a crystalline compound melting at $65 \cdot 5^{\circ}$.

Triphenylethylsilicane, $\mathrm{Ph}_{3} \mathrm{EtSi}$, and triphenylmethylsilicane,

$$
\mathrm{Ph}_{3} \mathrm{M} \mathrm{MSi} \text {, }
$$

were also prepared from triphenylsilicyl chloride.

\section{Benzylethylpropylsilicyl Oxide, (BzEtPrSi $)_{2} \mathrm{O}$.}

When benzylethylpropylsilicyl chloride is decomposed with water it yields a mixture of benzylethylpropylsilicol (Trans., 1907, 91, 726) and benzylethylpropylsilicyl oxide; the two compounds are easily separated by fractional distillation under reduced pressure $(25 \mathrm{~mm}$.). the silicol boiling at about $155^{\circ}$, the oxide at about $256^{\circ}$. A sample of the oxide boiling at $271-272^{\circ}(50 \mathrm{~mm}$.) was analysed :

0.2403 gave $0.6313 \mathrm{CO}_{2}$ and $0.2079 \mathrm{H}_{2} \mathrm{O} . \mathrm{C}=71 \cdot 6 ; \mathrm{H}=9 \cdot 6$.

$\mathrm{C}_{24} \mathrm{H}_{38} \mathrm{OSi}_{2}$ requires $\mathrm{C}=72 \cdot 2 ; \mathrm{H}=9.5$ per cent.

Benzylethylpropylsilicyl oxide is a rather viscid, colourless, almost odourless liquid, insoluble in water, but miscible with the ordinary organic solvents.

\section{Sulphonation of Benzylethylpropylsilicyl Oxide with Sulphuric Acid.}

When benzylethylpropylsilicyl oxide in quantities of about 10 grams at a time is treated with $1 \frac{1}{2}-2$ volumes of sulphuric acid only a slight rise in temperature occurs; on heating quickly and shaking vigorously a very viscous, homogeneous fluid results, and by the time the temperature has risen to $70^{\circ}$ or $80^{\circ}$ sulphonation is complete, as shown by the solubility of the product in a moderately large quantity of water. The rapidity with which sulphonation occurs seems to be rather greater than in the case of the corresponding silicol, as was perhaps to be expected.

In the hope of isolating a crystalline ammonium salt, the aqueous solution of the acid was neutralised with ammonia, the ammonium sulphate separated by the method previously described (loc. cit., p. 225), and the dried syrupy ammonium salt of the sulphonic acid dissolved in methyl alcohol; on adding a large volume of ethyl acetate to this solution, practically the whole of the dissolved salt was precipitated as a buttery mass, and the mother liquor did not contain any appreciable quantity of dissolved matter; this behaviour is very different from that of the crude ammonium salt obtained from the sulphonation product of benzylethylpropylsilicol; moreover, fractional 
precipitation of the buttery ammonium salt with ethyl acetate, from its solution in methyl alcohol, failed to alter its character appreciably, so that no crystallised ammonium sulphobenzylethylpropylsilicyl oxide could be isolated. This result seemed to show that the sulphonation products of the silicol and of the oxide were entirely different, but as a matter of fact they both contain sulphobenzylethylpropylsilicyl oxide, and the great difference in behaviour of the erude ammonium salts must be attributed to the accompanying by-products; why the latter should be so different it is difficult to conjecture. This method of purification having failed, the crude ammonium salt was treated in aqueous solution with $l$-menthylamine hydrochloride, and the oily precipitate of menthylamine salt separated; this was fractionally crystallised from ice-cold aqueous acetone, from which a considerable proportion was deposited in a flocculent condition, the remainder as an oil. After many recrystallisations, employing aqueous acetone, aqueous methyl alcohol, or wet ethyl acetate, some of the menthylamine salt was obtained in opaque nodules melting, when previously dehydrated, at about $215-220^{\circ}$; the further purification of this product proved to be very troublesome, but when systematically recrystallised, using aqueous acetone and wet ethyl acetate alternately for each series of operations, it finally yielded a well-defined, crystalline menthylamine salt melting at about $230^{\circ}$.

The details of these operations are omitted, but it may be noted that the great difficulty met with in isolating this salt is doubtless due to the presence of an isomeride which has not only a most baneful offect in preventing crystallisation, but which also remains very tenaciously in the crystalline deposits; when a considerable proportion of this isomeride is present, the mixture of salts is readily soluble in cold, wet ethyl acetate, whereas the pure compound melting at about $230^{\circ}$ is practically insoluble.

The menthylamine salt (m. p. 230 ) obtained in this way from the oxide resembled $l$-menthylamine sulphobenzylethylpropylsilicyl oxide (loc. cit. pp. 229, 729) in appearance, and like the latter erystallised with four molecules of water; a mixture of the two salts melted at the same temperature as its components:

0.2105 dried in the air lost $0.0165 \mathrm{H}_{2} \mathrm{O}$ at $100^{\circ} . \mathrm{H}_{2} \mathrm{O}=7 \cdot 8$.

$\mathrm{C}_{44} \mathrm{H}_{80} \mathrm{O}_{7} \mathrm{~N}_{2} \mathrm{~S}_{2} \mathrm{Si}_{2}+4 \mathrm{H}_{2} \mathrm{O}$ requires $\mathrm{H}_{2} \mathrm{O}=7 \cdot 65$ per cent.

$0 \cdot 1662$ gave $0.3682 \mathrm{CO}_{2}$ and $0 \cdot 1377 \mathrm{H}_{2} \mathrm{O} . \quad \mathrm{C}=60 \cdot 4 ; \mathrm{H}=9 \cdot 2$.

$0 \cdot 1925, \quad 0.4267 \mathrm{CO}_{2}, 0 \cdot 1598 \mathrm{H}_{2} \mathrm{O} . \quad \mathrm{C}=60 \cdot 4 ; \mathrm{H}=9 \cdot 2$. $\mathrm{C}_{44} \mathrm{H}_{80} \mathrm{O}_{7} \mathrm{~N}_{2} \mathrm{~S}_{2} \mathrm{Si}_{2}$ requires $\mathrm{C}=60 \cdot 8 ; \mathrm{H}=9 \cdot 2$.

The equivalent of the salt was also determined, the value obtained being $437 \cdot 2$ instead of $434 \cdot 4$ required by theory. 
The specific rotation was observed in methyl-alcoholic solution; 0.3558 , made up to 25 c.c. in a 2 -dcm. tube, gave $a-0.49^{\circ}$, whence $[a]_{\mathrm{D}}-17 \cdot 1^{\circ}$.

All these results agree with those required for the menthylamine salt of a disulphonic acid derived from the oxide ( $\mathrm{SiEtPrBz})_{2} \mathrm{O}$, and if the identity of this salt with that previously described is established, little doubt will remain as to the constitution of the latter.

Now in one respect the menthylamine salt isolated from the sulphonation product of the oxide appeared to differ from that obtained directly from pure ammonium sulphobenzylethylpropylsilicyl oxide; it did not seem to crystallise quite so readily or to form such large crystals. $^{*}$ For this reason some of the $l$-menthylamine salt prepared from the oxide was converted into sodium salt, and from the latter the cinchonidine and $d$-bornylamine salts were prepared; the cinchonidine salt melted at $148-150^{\circ}$ and its specific rotation in methyl alcohol was $[\alpha]_{\mathrm{D}}-71 \cdot 1^{\circ}$, values agreeing closely with those of the cinchonidine sulphobenzylethylpropylsilicyl oxide previously prepared from the silicol (loc. cit., p. 232); the properties of the bornylamine salt also agreed with those of the corresponding bornylamine salt from the silicol. There can be no doubt therefore that the silicol and the oxide yield one and the same acid when sulphonated under similar conditions, and that the sulphonic acid which has been resolved is in fact a derivative of the oxide. The by-products or other acids formed during these sulphonations have not yet been isolated.

\section{Sulphonation of Benzylethylpropylsilicyl Oxide with Chlorosulphonic Acid.}

The use of chlorosulphonic acid for the sulphonation of benzylmethylethylpropylsilicane gave results so much better than those obtained with sulphuric acid that it seemed advisable to try the action of the first-named agent on benzylethylpropylsilicyl oxide.

Chlorosulphonic acid (2 mols.) diluted with chloroform was added to a well-cooled solution of the oxide $(1 \mathrm{~mol}$.) in about five volumes of chloroform ; sulphonation occurred rapidly, with evolution of hydrogen chloride, and the solution was then immediately poured into water, and the chloroform distilled in steam; the clear aqueous solution of the acid was neutralised with ammonia, treated with excess of $l$-menthylamine hydrochloride, and the oily menthylamine salt separated and

* This difference between the two salts is possibly caused by some slight difference in the relative quantities of their components. The salt prepared directly from the ammonium salt contains equal quantities of $d A l B$ and $l A l B$, whilst that finally obtained from the oxide may contain unequal quantities of these components, partial resolution having occurred as a result of repeated fractional crystallisation. 
washed; it soon solidified, and was then fractionally crystallised from aqueous acetone. The several deposits were further treated systematically with moist ethyl acetate and acetone, alternately, and finally a menthylamine salt, melting at about $230^{\circ}$, was isolated.

This preparation was proved to be identical with the compound obtained by sulphonating the oxide with sulphuric acid; the dehydrated salt was analysed:

$$
\begin{aligned}
& 0.2044 \text { gave } 0.4532 \mathrm{CO}_{2} \text { and } 0.1705 \mathrm{H}_{2} \mathrm{O} . \mathrm{C}=60.5 ; \mathrm{H}=9 \cdot 3 \text {. } \\
& 0.1661 \Rightarrow 0.3675 \mathrm{CO}_{2}, 0.1409 \mathrm{H}_{2} \mathrm{O} . \quad \mathrm{C}=60.3 ; \mathrm{H}=9 \cdot 4 \text {. } \\
& 0.2133 \text { \# } 0.4742 \mathrm{CO}_{2} \text {, } 0 \cdot 1848 \mathrm{H}_{2} \mathrm{O} . \quad \mathrm{C}=60 \cdot 6 ; \mathrm{H}=9 \cdot 6 \text {. } \\
& \mathrm{C}_{44} \mathrm{H}_{80} \mathrm{O}_{7} \mathrm{~N}_{2} \mathrm{~S}_{2} \mathrm{Si}_{2} \text { requires } \mathrm{C}=60 \cdot 8 ; \mathrm{H}=9 \cdot 2 \text { per cent. }
\end{aligned}
$$

The isolation of a pure menthylamine salt from the product just described seemed to offer less difficulty than when sulphuric acid was used as the sulphonating agent, but as the two series of experiments were carried out at rather distant intervals, the behaviour of the two crude preparations was not directly compared.

\section{Sulphonation of Phenylbenzylethylpropylsilicane with Chlorosulphonic Acid.}

Since phenylbenzylethylpropylsilicane is hydrolysed by sulphuric acid with separation of benzene (loc. cit., p. 223), some experiments were made to see whether hydrolysis could be avoided, and a monosulphonic derivative of the silico-hydrocarbon obtained by using chlorosulphonic acid.

Pure phenylbenzylethylpropylsilicane was dissolved in chloroform, and chlorosulphonic acid ( $1 \mathrm{~mol}$.), also dissolved in chloroform, was slowly added, cooling in ice: towards the end of the operation hydrogen chloride was evolved, but on testing a small quantity of the solution it was found to contain a large proportion of an oil which was insoluble in water. A chloroform solution of chlorosulphonic acid $(1 \mathrm{~mol}$.) was then slowly run in, testing from time to time, but it was not until the whole of this had been added that the sulphonation was complete. These observations seemed to show that the silicohydrocarbon was first decomposed by the chlorosulphonic acid, probably in accordance with the following equation: $\mathrm{SiPhBzEtPr}+$ $\mathrm{SO}_{3} \mathrm{HCl}=\mathrm{SiBzEtPrCl}+\mathrm{C}_{6} \mathrm{H}_{5} \cdot \mathrm{SO}_{3} \mathrm{H}$.

The chloroform having been distilled in steam, the clear aqueous solution was neutralised with ammonia, and treated with excess of $l$-menthylamine hydrochloride: the precipitated menthylamine salt soon solidified and was repeatedly crystallised, using first aqueous acetone, then anhydrous acetone, and finally wet ethyl acetate; from the last-named solvent the salt was ultimately obtained in colourless, lustrous, hydrated crystals melting at about $230^{\circ}$. 
The anhydrous salt was analysed :

$0 \cdot 1888$ substance gave $0.4192 \mathrm{CO}_{2}$ and $0.1573 \mathrm{H}_{2} \mathrm{O} . \mathrm{C}=60 \cdot 5 ; \mathrm{H}=9 \cdot 3$. $0 \cdot 1628 \quad$ " $, 0.3625 \mathrm{CO}_{2}, 0.1376 \mathrm{H}_{2} \mathrm{O} . \mathrm{C}=60 \cdot 7 ; \mathrm{H}=9 \cdot 4$.

These results agree with those $(\mathrm{C}=60 \cdot 8 ; \mathrm{H}=9 \cdot 2)$ required for the menthylamine salt derived from benzylethylpropylsilicyl oxide, and the identity of this preparation with the latter was completely established. The action of chlorosulphonic acid on the silico-hydrocarbon, therefore, is similar to that of sulphuric acid as regards the elimination of the phenyl radicle.

\section{Benzylethyldipropylsilicane.}

In the preparation of benzylethylpropylsilicyl chloride from molecular proportions of benzylethylsilicon dichloride and magnesium propyl bromide, a considerable proportion of the dichloride seems to be unchanged (loc. cit., p. 722); after fractionally distilling the product many times, and separating the benzylethylpropylsilicyl chloride and compounds of higher boiling point, there remains a liquid which passes over from about $180-190^{\circ}$, and which is doubtless a mixture of the dichloride and its propyl derivative.

This material was utilised for the preparation of benzylethyldipropylsilicane, and for this purpose it was treated with excess of magnesium propyl bromide in the usual way; in order to ensure complete interaction the ether was then distilled and the pasty mass heated in an oil-bath at $130^{\circ}$ for about two hours. After cooling and dissolving the magnesium salts in water, the oily product was separated and submitted to systematic fractional distillation. The liquid finally collected at $178^{\circ}(50 \mathrm{~mm}$.) was redistilled under atmospheric pressure and the portion boiling at $280^{\circ}$ was analysed :

0.1708 gave $0.4710 \mathrm{CO}_{2}$ and $0.1729 \mathrm{H}_{2} \mathrm{O} . \mathrm{C}=75 \cdot 2 ; \mathrm{H}=11 \cdot 2$. $\mathrm{C}_{15} \mathrm{H}_{26} \mathrm{Si}$ requires $\mathrm{C}=76.8 ; \mathrm{H}=11 \cdot 1$ per cent.

Benzylethyldipropylsilicane is a colourless, mobile, highly refractive liquid, very similar to benzylmethylethylpropylsilicane and other homologues which bave already been described.

\section{Sulphonation of Benzylethyldipropylsilicane with Chlorosulphonic Acid.}

The sulphonic derivative of benzylethyldipropylsilicane was obtained very easily by treating the silico-hydrocarbon with chlorosulphonic acid; the operation was carried out in chloroform solution, and the acid then converted into its ammonium salt exactly as described in the preparation of ammonium benzylmethylethylpropylsilicanesulphonate (loc. cit., p. 739.) The ammonium salt was next treated with 
$l$-menthylamine hydrochloride in aqueous solution, and the colourless, solid precipitate separated by filtration.

\section{1-Menthylamine Benzylethyldipropylsilicanesulphonate, $\mathrm{SiEtPr} \cdot \mathrm{CH}_{2} \cdot \mathrm{C}_{6} \mathrm{H}_{4} \cdot \mathrm{SO}_{3} \mathrm{H}, \mathrm{C}_{10} \mathrm{H}_{19} \cdot \mathrm{NH}_{2}$.}

The crude salt is readily soluble in wet light petroleum, but separates on cooling in lustrous, rather poorly defined plates; after repeated crystallisation from this solvent it is obtained in a pure condition, a small quantity of some oily by-product passing into the mother liquors.

A sample of the salt dried in the air until constant was heated at $100^{\circ}$ :

0.2240 lost $0.0159 \mathrm{H}_{2} \mathrm{O} . \quad \mathrm{H}_{2} \mathrm{O}=7 \cdot 1$. $\mathrm{C}_{25} \mathrm{H}_{47} \mathrm{O}_{3} \mathrm{NSSi}+2 \mathrm{H}_{2} \mathrm{O}$ requires $\mathrm{H}_{2} \mathrm{O}=7 \cdot 1$ per cent.

An analysis of the anhydrous salt gave the following result:

0.1143 gave $0.2667 \mathrm{CO}_{2} ; 0.1050 \mathrm{H}_{2} \mathrm{O} . \mathrm{C}=63.6 ; \mathrm{H}=10.2$.

$\mathrm{C}_{25} \mathrm{H}_{47} \mathrm{O}_{3} \mathrm{NSSi}$ requires $\mathrm{C}=63.9 ; \mathrm{H}=10.0$ per cent.

The equivalent of the salt was also determined by the method previously used; the value obtained was 470.8 instead of 469.4 required by theory.

The specific rotation was determined in methyl-alcoholic solution :

0.589 , made up to 25 c.c. in a 2 -dcm. tube, gave $a-0.70^{\circ}$, whence $[a]_{\mathrm{D}}-14 \cdot 9^{\circ}$.

Anhydrous menthylamine benzylethyldipropylsilicanesulphonate melts at $135^{\circ}$, the hydrated compound at $62.5-63^{\circ}$; it is practically insoluble in water, but very readily soluble in all the ordinary organic solvents, including dry light petroleum; a concentrated solution of the salt in this solvent does not yield crystals when kept over sulphuric acid, but on adding a little water, the hydrated compound separates almost immediately on the water globules, and when the petroleum is thoroughly wetted almost the whole of the salt is quickly deposited.

In this respect, in outward properties, and in general behaviour this salt closely resembles the corresponding derivative of $d l$-benzylmethylethylpropylsilicanesulphonic acid (loc. cit., p. 735), so closely, in fact, that the latter can hardly be regarded as a definite partially racemic compound. The specific rotation of the salt of the dipropyl derivative is also very nearly the same as that of the derivative of the externally compensated acid, which was found to be $[a]_{D}-15^{\circ}$ in methyl-alcoholic solution. 


\section{Cinchonidine Benzylethyldipropylsilicanesulphonate.}

The pure menthylamine salt was decomposed with the theoretical quantity of sodium carbonate, the menthylamine distilled in steam, and the resulting sodium salt treated with cinchonidine hydrochloride, $\left(\mathrm{C}_{19} \mathrm{H}_{22} \mathrm{ON}_{2}, \mathrm{HCl}+2 \mathrm{H}_{2} \mathrm{O}\right)$, in aqueous solution; the precipitated cinchonidine salt was then recrystallised several times from aqueous alcohol, from which it separated in lustrous prisms melting at $191-192^{\circ}$. The salt is readily soluble in most of the ordinary organic solvents, but only sparingly so in cold ethyl acetate or ether, and practically insoluble in light petroleum or water.

Its specific rotation was determined in methyl-alcoholic solution :

0.4797 , made up to 25 c.c. in a 2 -dcm. tube, gave $\alpha-2 \cdot 66^{\circ}$, whence $[a]_{D}-69 \cdot 3^{\circ}$.

The resemblance between this salt and the corresponding derivative of the $d l$-acid (loc. cit., p. 743) is extremely close, the latter melting at $194-195^{\circ}$, and having a specific rotation $[a]_{\mathrm{D}}-72^{\circ}$.

\section{Cinchonidine Hydrogen Benzylethyldipropylsilicanesulphonate.}

This salt was obtained by treating the normal salt with excess of hydrochloric acid in methyl-alcoholic solution and then precipitating with water ; the oily product soon solidified, and was then recrystallised from aqueous acetone. It crystallises in needles, melting and decomposing at $222-224^{\circ}$, and resembles the corresponding salt of the $d l$-acid in its behaviour towards solvents.

\section{Quinine Benzylethyldipropylsilicanesulphonate.}

The addition of a solution of quinine hydrochloride to a solution of the sodium salt caused the precipitation of an oil which soon solidified. The precipitate was washed well with water and crystallised from a mixture of ethyl acetate and light petroleum until a constant melting point was attained.

The salt formed long, colourless, lustrous needles, melted sharply at $175^{\circ}$, and was readily soluble in ethyl acetate, alcohol, acetone, ether, or benzene, but practically insoluble in light petroleum or water.

Its specific rotation was determined in methyl-alcoholic solution :

$0 \cdot 216$, made up to 25 c.c. in a 2 -dem. tube, gave a $-1.94^{\circ}$, whence $[a]_{\mathcal{D}}-112 \cdot 3^{\circ}$. 


\section{Quinine Hydrogen Benzylethyldipropylsilicanesulphonate.}

This compound was obtained from the normal salt in the usual manner and crystallised from aqueous methyl alcohol, from which it separated in long, colourless needles melting at $223-224^{\circ}$. It was soluble in chloroform, but practically insoluble in ethyl acetate, anhydrous acetone, or ether. This, and the normal quinine salt, were very similar to the corresponding derivatives of the $d l$-acid (loc. cit., p. 742).

\section{Diphenylethylsilicyl Chloride, $\mathrm{SiPh}_{2} \mathrm{EtCl}$.}

The interaction of ethylsilicon trichloride and magnesium phenyl bromide has already been described (loc. cit., p. 215), and it has been stated that after isolating the main product, phenylethylsilicon dichloride, by fractional distillation there remains a considerable quantity of a dark yellow or brown oil boiling above $240^{\circ}$ (atmospheric pressure). This by-product, accumulated from many preparations, was submitted to systematic fractional distillation under reduced pressure, using a rod and disk column, and ultimately, after separating the diphenyl, three silicon compounds were isolated from it, namely, diphenylethy] silicyl chloride, triphenylsilicyl chloride, and triphenylsilicol.

Diphenylethylsilicyl chloride is a fuming, mobile liquid, boiling at about $240^{\circ}\left(115 \mathrm{~mm}\right.$.) and at $206-208^{\circ}(50 \mathrm{~mm}$.) ; the freshlydistilled liquid was colourless, but after a short time some of the fractions became pink, some green, probably owing to traces of impurity. The fraction collected at $206-208^{\circ}(50 \mathrm{~mm}$.), after many distillations under different pressures, did not alter in boiling point appreciably when re-distilled, and on analysis :

0.9329 gave $0.5524 \mathrm{AgCl}$. $\mathrm{Cl}=14 \cdot 6$.

A volumetric estimation of the chlorine in the same sample gave : $\mathrm{Cl}=14 \cdot 1$.

$$
\mathrm{C}_{14} \mathrm{H}_{15} \mathrm{ClSi} \text { requires } \mathrm{Cl}=14 \cdot 4 \text { per cent. }
$$

Diphenylethylsilicyl chloride reacts readily with $p$-toluidine in light petroleum solution, but the product could not be separated from the $p$-toluidine hydrochloride without adding water, the result being that the toluidide was completely decomposed.

\section{Diphenylethylsilicyl Oxide, $\left(\mathrm{Ph}_{2} \mathrm{EtSi}\right)_{2} \mathrm{O}$.}

Diphenylethylsilicol, like benzylethylpropylsilicol (loc. cit., p. 726) and phenylethylpropylsilicol (loc. cit., p. 219), shows a great tendency to pass into the corresponding oxide, and up to the present no 
preparation giving satisfactory analytical results has been obtained. When diphenylethylsilicyl chloride is decomposed with water, the resulting oil extracted, and kept over sulphuric acid, a crystalline substance is deposited in the course of a few days. This product is the oxide; it separates from aqueous methyl alcohol in well-defined, colourless, rhomboidal plates melting at $65-66^{\circ}$, and is readily soluble in the common alcohols, but only moderately so in cold chloroform, ether, light petroleum, or benzene :

$$
\begin{gathered}
0.1487 \text { gave } 0.4141 \mathrm{CO}_{2} \text { and } 0.0927 \mathrm{HO}_{2} . \quad \mathrm{C}=76.0 ; \mathrm{H}=6.9 . \\
0.1591 \quad 0.4446 \mathrm{CO}_{2}, 0.0980 \mathrm{H}_{2} \mathrm{O} . \quad \mathrm{C}=76.2 ; \mathrm{H}=6.8 . \\
\mathrm{C}_{28} \mathrm{H}_{30} \mathrm{OSi}_{2} \text { requires } \mathrm{C}=76.6 ; \mathrm{H}=6.8 \text { per cent. }
\end{gathered}
$$

The conditions under which the silicol passes into the oxide and vice versa have not yet been studied.

\section{Triphenylsilicyl Chloride, $\mathrm{Ph}_{3} \mathrm{SiCl}$.}

The fractions of the crude by-product (p. 207) collected from about $260-330^{\circ}(50 \mathrm{~mm}$.) solidified more or less completely, and the crystalline deposit was separated by filtration and then washed with light petroleum in a stream of dry air. After several crystallisations from boiling light petroleum, in which the substance was readily solut.le, it was obtained in colourless prisms melting at $110-111^{\circ}$. This product did not fume appreciably in the air, but as it was readily decomposed by water, it was necessary to exclude all moisture during its purification; it gave an immediate precipitate of silver chloride when added to an alcoholic solution of silver nitrate, and on exposure to the air it underwent decomposition, giving, after many days' exposnre, a sample of triphenylsilicol melting at about $145-147^{\circ}$.

The following analysis of the compound and its behaviour towards water showed that it was triphenylsilicyl chloride :

0.2507 gave (volumetrically) $0.0303 \mathrm{Cl} . \mathrm{Cl}=12 \cdot 1$.

$$
\mathrm{C}_{18} \mathrm{H}_{15} \text { ClSi requires } \mathrm{Cl}=12 \cdot 0 \text { per cent. }
$$

The occurrence of this compound in the product of the interaction of ethylsilicon trichloride and magnesium phenyl bromide is possibly due to the presence of silicon tetrachloride as impurity in the ethyl derivative used, but as the latter had been carefully fractionated, and as the relative quantity of the triphenyl derivative was considerable, it may be that the ethyl is displaced by the phenyl group during the interaction.

It may be noted that triphenylsilicyl chloride was first obtained in small quantities by Kipping and Lloyd (Trans., 1901, 79, 449), but the melting point given by them is far too low; unless there is plenty of material at disposal for recrystallisation, it is difficult to 
avoid some slight decomposition by atmospheric moisture, and the melting point then rapidly falls.

\section{Triphenylsilicol, $\mathrm{Ph}_{3} \mathrm{SiOH}$.}

The fractions of the by-product (p. 207) collected from about $330-380^{\circ}(50 \mathrm{~mm}$.) also solidified partly, owing to the deposition of a compound free from halogen; this was separated and recrystallised from a mixture of ether and light petroleum, from which it was obtained in lustrous prisms melting at $150.5-151.5^{\circ}$ (uncorrected):

0.2099 gave $0.6042 \mathrm{CO}_{2}$ and $0.1142 \mathrm{H}_{2} \mathrm{O} . \mathrm{C}=78.5 ; \mathrm{H}=6.0$. $\mathrm{C}_{18} \mathrm{H}_{16} \mathrm{OSi}$ requires $\mathrm{C}=78.2 ; \mathrm{H}=5.8$ per cent.

This compound was identified as triphenylsilicol, the presence of which might naturally be ascribed to the unavoidable action of atmospheric moisture on the corresponding chloride during distillation, \&c., but as it was obtained in such large relative quantities its occurrence may possibly be due to the decomposition of the ether used in the Grignard reaction.

The melting point of triphenylsilicol has been variously given as 139-141 ${ }^{\circ}$ (Polis, Ber., 1886, 19, 1019), $148^{\circ}$ (Kipping and Lloyd, loc. cit.), $155^{\circ}$ (Dilthey and Eduardoff, Ber., 1904, 37, 1139), $148-150^{\circ}$ (Ladenburg, Ber., 1907, 40, 2274); a sample of the silicol prepared from the chloride was repeatedly crystallised from a mixture of ether and light petroleum until three successive fractions melted simultaneously; this preparation had the melting point given above $\left(150 \cdot 5-151 \cdot 5^{\circ}\right)$.

\section{Triphenylethylsilicane, $\mathrm{SiPh}_{3} \mathrm{Et}$.}

This silico-hydrocarbon was prepared by treating triphenylsilicyl chloride with excess of magnesium ethyl bromide; as no rise in temperature occurred on mixing the reagents, the ether was distilled off, and the residue heated in an oil-bath at $130^{\circ}$ for three hours. After cooling and adding water, the product, which separated as a colourless solid, was crystallised several times from light petroleum. The yield was practically theoretical :

$0 \cdot 1771$ gave $0.5390 \mathrm{CO}_{2}$ and $0.1113 \mathrm{H}_{2} \mathrm{O} . \quad \mathrm{C}=83.0 ; \mathrm{H}=7 \cdot 0$. $\mathrm{C}_{20} \mathrm{H}_{20} \mathrm{Si}$ requires $\mathrm{C}=83.2 ; \mathrm{H}=6.9$ per cent.

Triphenylethylsilicane crystallises in rhomboidal prisms melting at $76^{\circ}$, and is readily soluble in light petroleum, ether, chloroform, benzene, ethyl acetate, or acetone, only moderately so in alcohol, and insoluble in water.

This silico-hydrocarbon seems to have been obtained recently by 
Ladenburg* (Ber., 1907, 40, 2274), together with triphenylsilicane, by heating triphenylsilicyl bromide with zinc ethyl; according to Ladenburg, the melting point is $72-74^{\circ}$.

\section{Triphenylmethylsilicane, $\mathrm{SiPh}_{3} \mathrm{Me}$.}

This compound was prepared from triphenylsilicyl chloride and magnesium methyl iodide in a similar manner, heating at $130^{\circ}$ for three hours. It was purified by recrystallisation from light petroleum, a theoretical yield being obtained :

0.1773 gave $0.5389 \mathrm{CO}_{2}$ and $0.1053 \mathrm{H}_{2} \mathrm{O} . \mathrm{C}=82.9 ; \mathrm{H}=6.6$. $\mathrm{C}_{19} \mathrm{H}_{18} \mathrm{Si}$ requires $\mathrm{C}=83.1 ; \mathrm{H}=6.6$ per cent.

This compound melts at $67-67 \cdot 5^{\circ}$, but otherwise it resembles the ethyl derivative very closely.

Government grants, for which the authors are greatly indebted to the Committee of the Royal Society, have been used to cover a portion of the cost of this investigation.

UNiversity College, NotiINGHAM. 